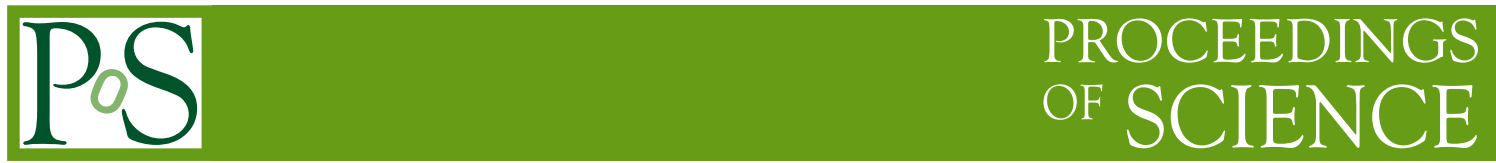

\title{
Short Orbital Period Cataclysmic Variables
}

\author{
Sergey Zharikov* \\ ${ }^{1}$ Observatorio Astronomico Nacional, Instituto de Astronomía, Universidad Nacional Autónoma \\ de México, Ensenada, BC, México \\ E-mail: zhareastrosen. unam.mx
}

Cataclysmic Variables (CVs) are semi-detached binary systems consisting red/brown dwarfs filling its corresponding Roche lobe and loosing matter onto white dwarf (WD) companions. Classification of CVs is based on behavior of outbursts. In this report, we review properties of Short Orbital Period Cataclysmic Variables (77 min $<P_{\text {orb }}<120 \mathrm{~min}$ ). In mentioned orbital period range three subclasses of CVs were distinguished: SU UMa-, ER UMa- and WZ Sge-type objects. WZ Sge-type objects more probably includes also systems evolved past of the period minimum. The last are formed so-called bounce-backs or post-period minimum systems. Main characteristics of WDs, donor stars and accretion disks in the Short Orbital Period Cataclysmic Variables are discussed.

The Golden Age of Cataclysmic Variables and Related Objects - III, Golden2015

7-12 September 2015

Palermo, Italy

${ }^{*}$ Speaker. 


\section{Introduction}

Cataclysmic Variables (CVs) are semi-detached binary systems consisting a red/brown dwarf (secondary) filling its Roche lobe and loosing matter to a white dwarf companion (primary). The dwarf nova (DN) subclass of CVs show outburst behavior when system's brightness increases until 2-8 mag during days to weeks. The actual classification of CVs is based, in general, on characteristics of outbursts. Figure 1, (left) illustrates the current concept of evolution and generally accepted nomenclature of CVs. Below we discuss main characteristics of CVs with orbital periods below the period gap ( $\sim 2-3$ hours).
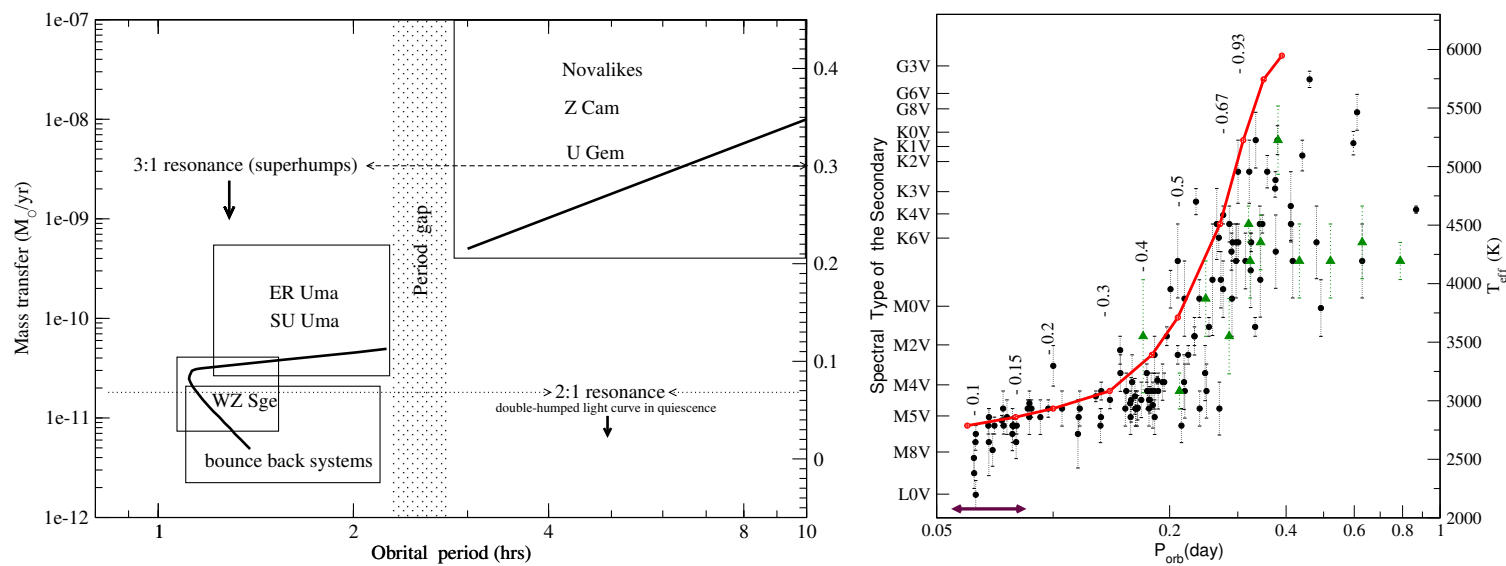

Figure 1: Left) The current concept of evolution of CVs (solid line) are shown at the mass transfer rate / the mass ratio vs. the orbital period diagram. Right) The distribution of CVs on the spectral class / effective temperature vs. orbital period diagram. The filled circle are CVs and triangles are low mass X-ray binaries. The solid line shows Sp. Type - period relation for ZAMS stars and numbers corresponds to masses of ZAMS secondary. The arrow marks the range of periods which discussed here.

During evolution orbital periods of CVs decrease. The observed period minimum of CVs with hydrogen-rich secondaries is about $77 \mathrm{~min}$ [1] and is caused by a response of the donor star to ongoing mass loss [2]. It is predicted a significant increase in a number of CVs at smaller periods with a spike close to the period minimum [3]. After reach the period minimum CVs should evolve to back toward longer periods and form so-called bounce-back systems. Actually, we distinguish three commonly accepted types of CVs in the period range of 77 - 120 min: SU UMa-, ER UMa-, and WZ Sge-type objects. The last more probably includes also bounce back systems. There are also magnetic CVs (polars) and some nova-like systems in this period range but they are no subject of this review.

\section{Geometry of Short Orbital Period CVs. Primaries and Secondaries}

Figure 2 illustrates the geometry of CVs below the period gap. The mean mass of WDs in these systems is $\sim 0.8 \mathrm{M}_{\odot}[4,5,6]$. It is significantly higher that expected $\left(\sim 0.5 \mathrm{M}_{\odot}\right)$ from the $\mathrm{CV}$ formation scenario [7]. Two mechanisms were proposed to explain the excess. The first is a short phase of thermal time-scale mass transfer in the beginning of $\mathrm{CV}$ formation during which WDs gain a sufficient amount of mass. The other is the growing of the WD mass during the CV 

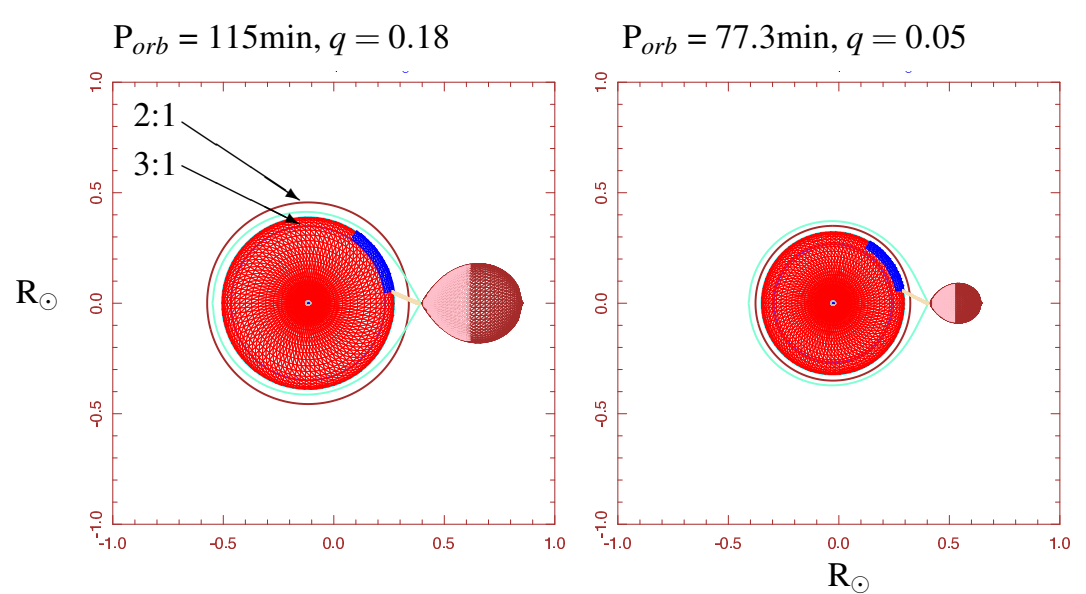

$\mathrm{P}_{\text {orb }}=89.0 \mathrm{~min}, q=0.03$

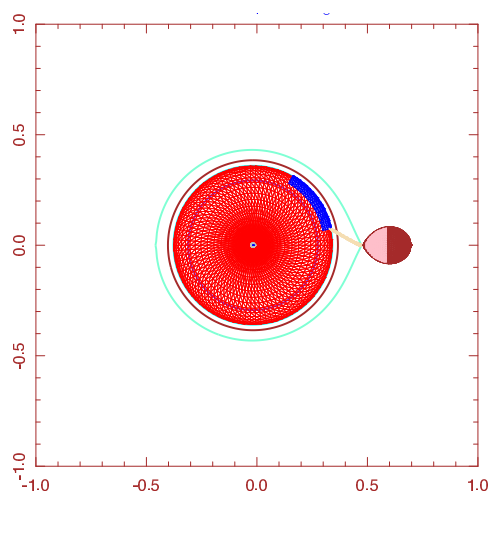

Figure 2: The illustration of the geometrical size of Short Period CVs $\left(M_{1}=0.8 M_{\odot}\right)$ : left $)$ at the lower boundary of the period gap; center) at the period minimum; right) a bounce back system. The size of the accretion disk corresponds to the tidal radius $\left(r_{d} / a=0.60 /(1+q)\right)$, the $3: 1$ and $2: 1$ resonance radii are marked on the left panel.

evolution [8]. WDs in the short-period CVs are also hotter (10000-20000 K, [9]) than expected for their age. This provides an evidence for some thermal impact of prolonged accretion onto WDs [10]. Measured masses of the secondaries are in the range of $\sim 0.05-0.15 \mathrm{M}_{\odot}$. The mass ratio $q=M_{2} / M_{1}$ is always below 0.2 [11] and the $3: 1$ resonance radius $\left(R_{3: 1} / a=(1 / 3)^{2 / 3}(1+q)^{-1 / 3}\right)$ is inside of the primary Roche Lobe. The spectral class of donor stars (before the period minimum) is about M5 $( \pm 1) \mathrm{V}$ (Fig. 1, right), their effective temperature is in the range of $2800-3200 \mathrm{~K}$. We note, however, that there exist only about 25 classifications of secondaries from optical/infrared spectroscopy. Most of others are based on the relationship between the orbital period and secondary Roche lobe radius and the mass-radius relationship for ZAMS stars (the solid line in Figure 1, right). Nevertheless, there are evidences that majority of donor stars in CVs have radii about 10 per cent larger than predicted [12]. It implies evolved donor stars in those CVs. Only at the period minimum effective temperatures of secondaries decrease and correspond to brown dwarfs. With decreasing of the orbital period mass ratio also decreases and, for systems with $q<0.08$, the 2:1 resonance radii $\left(R_{2: 1} / a=(1 / 3)^{2 / 3}(1+q)^{-1 / 3}\right)$ is also into the primary Roche lobe. The secondary become degenerated and its radius grows down to the Jupiter's size.

Recently some new objects (EI Psc, OZ Ser, CSS100531) were discovered with very hot donor stars ( $\sim \mathrm{K} 4 \mathrm{~V}$ spectral type) $[13,14,15]$. They probably form an additional subclass of CVs in the discussed period range.

\section{SU UMa-type stars}

SU UMa-type CVs are dwarf novae which show two types of outbursts, namely normal and superoutbursts. A typical normal outburst occurs every $\sim 10-100 \mathrm{~d}$ and has a duration of 3-5 d. Superoutbursts are $\sim 1$ mag brighter and $\sim 10$ times less frequent than normal outbursts. A unique property of superoutbursts is the appearance of superhumps, well-defined brightness modulations with a period of a few per cent longer than the orbital one. The origin of superhumps is basically explained as a result of varying tidal dissipation in an eccentric accretion disk, whose eccentricity is excited by the $3: 1$ orbital resonance. It is important also to note than the outer radius of the 
accretion disk in those systems may reach the tidal limitation radius during super-outbursts (see Fig. 2 and fig. 23 in [16]).

Most of observed non-magnetic CVs in the orbital period range of $78 \min \lesssim P_{\text {orb }} \lesssim 150 \min$ are SU UMa-type systems. For a comprehensive review of SU UMa-type stars and the observed superhump properties, see $[11,17,18,19]$.

\section{ER UMa-type stars}

ER UMa-type stars are a small subclass of CVs, which are characterized by the extremely high outburst frequency and short supercycles. For example, the prototype of the subclass ER UMa shows normal and superoutbursts every $4.4 \mathrm{~d}$ and $43 \mathrm{~d}$, respectively [20, 21]. ER UMa-type systems seem to be similar to the SU UMa-type stars with only higher activity and luminosity due to their higher mass transfer rates. The list of known ER UMa-type systems is given in Table 1. There are ER UMa-type objects practically at the period minimum. Patterson et al. (2013), based on observations of the nova-like system BL Lyn, which light curve in 2005 became indistinguishable from ER UMa-type objects, proposed that the ER UMa-systems are close to their previous nova explosions, implying that their putative high mass transfer rates are the result of irradiation of the secondary from the disk [22]. On the other hand, Kato et al. (2016) proposed that the exceptionally high mass-transfer rate in ER UMa-type objects may be a result of a stripped core evolved secondary in a system evolving towards AM CVn-type objects [23].

Table 1: Basic properties of ER UMa variables (from [24] and references therein).

\begin{tabular}{|l|c|c|c|c|c|c|c|c|}
\hline Star & $\begin{array}{c}P_{\text {orb }} \\
\text { days }\end{array}$ & $\begin{array}{c}P_{\text {sh }} \\
\text { days }\end{array}$ & $\begin{array}{c}\varepsilon \\
\%\end{array}$ & $\begin{array}{c}T_{s} \\
\text { days }\end{array}$ & $\begin{array}{c}T_{n} \\
\text { days }\end{array}$ & $\begin{array}{c}T_{\text {sup }} \\
\text { days }\end{array}$ & $\begin{array}{c}A_{\text {sup }} \\
\text { mag }\end{array}$ & $\begin{array}{c}A_{\mathrm{n}} \\
\text { mag }\end{array}$ \\
\hline RZ LMi & $?$ & 0.05946 & $?$ & 18.9 & 3.8 & 11 & 2.5 & 2.0 \\
DI UMa & 0.054564 & 0.0555 & 1.72 & 25.0 & 5.0 & 12 & 2.9 & 2.1 \\
ER UMa & 0.06366 & 0.065552 & 2.97 & 43.0 & 4.4 & 23 & 2.6 & 2.2 \\
V1159 Ori & 0.062178 & 0.064284 & 2.11 & $44.6-53.3$ & 4.0 & 16 & 2.2 & 1.4 \\
IX Dra & 0.06646 & 0.066968 & 0.76 & 54.0 & 3.1 & 16 & 2.2 & 1.7 \\
\hline
\end{tabular}

\section{WZ Sge-type stars}

WZ Sge-type stars are a subgroup of the dwarf novae, characterized by very long recurrence times (several years or even decades) of superoutbursts with very large amplitudes exceeding $6 \mathrm{mag}$, and the lack or great rarity of normal outbursts. Their other peculiar properties are the appearance of so-called early superhumps during the early stage of superoutbursts and multiple post-outbursts rebrightenings. The main characteristics of the prototype of the subclass (WZ Sge) are summarized below: the short orbital period of $81.6 \mathrm{~min}$, close to the predicted period minimum; the spectra in quiescence show the strong double-peaked Balmer emission lines from the accretion disk surrounded by broad absorptions, formed by the primary white dwarf (see for an example [25]); infrequent $\sim 20-30$ years and the large-amplitude $\left(\sim 8^{m} ; 1913,1946,1978,2001\right)$ superoutbursts succeeded by echo outbursts, there are not normal outbursts; optical light curves during 
Table 2: Parameters of WZ Sge and of the bounce back candidates.

\begin{tabular}{|l|lccccccc|}
\hline NN/Object & $\begin{array}{l}\mathrm{P}_{\text {orb }} \\
(\mathrm{days})\end{array}$ & $\begin{array}{c}\mathrm{V} \\
(\mathrm{mag})\end{array}$ & $\mathrm{q}$ & $\begin{array}{c}\mathrm{M}_{1} \\
\left(\mathrm{M}_{\odot}\right)\end{array}$ & $\begin{array}{c}\mathrm{M}_{2} \\
\left(\mathrm{M}_{\odot}\right)\end{array}$ & $\begin{array}{c}\mathrm{T}_{\text {eff }}^{W D} \\
(K)\end{array}$ & $\begin{array}{c}i \\
\left({ }^{o}\right)\end{array}$ & $\mathrm{LC}^{1}$ \\
\hline 1. WZ Sge & 0.0567 & $\sim 15$ & 0.092 & 0.85 & 0.078 & 13500 & 77 & $+\mathrm{s}$ \\
2. GW Lib & 0.0533 & 19.1 & 0.060 & 0.84 & 0.05 & & 11 & \\
3. V455 And & 0.0563 & 16.5 & $0.060 \mathrm{~s}$ & & $>\mathrm{M} 9$ & 11500 & 83 & $+\mathrm{q}$ \\
4. AL Com & 0.0567 & 19.1 & 0.060 & & & 16300 & & $+\mathrm{q}$ \\
5. SDSS1035 & 0.057 & 18.7 & $0.055 \mathrm{e}$ & 0.94 & 0.05 & 10100 & 83 & \\
6. V406 Vir & 0.056 & 17.8 & 0.05 & $\sim 1.0$ & 0.05 & 12000 & $\sim 70$ & $+\mathrm{q}$ \\
7. EZ Lyn & 0.059 & 17.8 & $0.05 \mathrm{~s}$ & $\sim 0.9$ & 0.045 & 13000 & $\sim 70$ & $+\mathrm{q}$ \\
8. EG Cnc & 0.060 & 18.8 & $0.035 \mathrm{~s}$ & & & 12300 & & $+\mathrm{s}$ \\
9. RX1050-14 & 0.062 & 17.6 & $<0.055 \mathrm{v}$ & & & 13000 & $<65$ & \\
10. GD552 & 0.0713 & 16.6 & $<0.052 \mathrm{v}$ & & $<0.08$ & 10900 & $<60$ & \\
11. RE1255 & 0.083 & 19.0 & $<0.064 \mathrm{v}$ & $>0.9$ & $<0.08$ & 12000 & $<5$ & - \\
\hline
\end{tabular}

${ }^{1}$ light curve (LC) features: "+" LC shows a double-hump during the orbital period;

"s" - during super-outbursts; "q" - during quiescence; "-" absent of double-humps in LCs.

Recent bounce-back candidates:

OT J075418.7+381225, OT J230425.8+062546 [35], SSS J122221.7-311523[36, 37]

a super-outburst show long-lasting super-humps [26]; there are double-humped in the light curves during super-outbursts and in quiescence [27, 26]; there are evidence of forming of spiral arms in the disk during super-outbursts [28, 29]; the accretion disc is asymmetric in quiescence, and the bright spot region is to be extended along the mass transfer stream [30, 31]; the outer layers of the accretion disc is a low density and a low temperature of $\sim 3000 \mathrm{~K}$ [32]; there is some evidence that a cavity was formed in the inner part of the disk during quiescence implying an annulus-shaped accretion disc [33].

The number of WZ Sge-type stars increases with decreasing orbital periods and they seem to be a continuation of the evolution of SU UMa-type stars when mass transfer rates begin to drop closely to the period minimum (Fig. 3). Indeed, optical spectra of these objects in quiescence often show Balmer emission lines from the accretion disk surrounded by broad absorption troughs formed in the high-density photosphere of the WD, This strongly indicates a low mass transfer rate and accordingly a very low accretion luminosity. Most of spectrally studied WZ Sge-type systems include massive $\left(0.8-1.0 \mathrm{M}_{\odot}\right)$ and relatively cool $(\sim 12000 \mathrm{~K})$ WDs. The distribution of the mass ratios of the stellar components in WZ Sge-type stars shows a sharp peak at $q \approx 0.07$, indicating a very low-mass secondary star in these systems, close or even below the hydrogenburning minimum mass limit. We also note that there exists a significant number of short-period $\mathrm{CVs}$, which have never been observed in outbursts, but whose optical spectra are indistinguishable from WZ Sge-type systems in quiescence. These objects can be considered as WZ Sge-type star candidates which outbursts were not yet recorded because of their faintness and insufficiently long observations. For a comprehensive review of WZ Sge-type stars see [34].

\section{Bounce-back systems}

The bounce-back systems (or period bouncers) are such CVs that have passed the period min- 

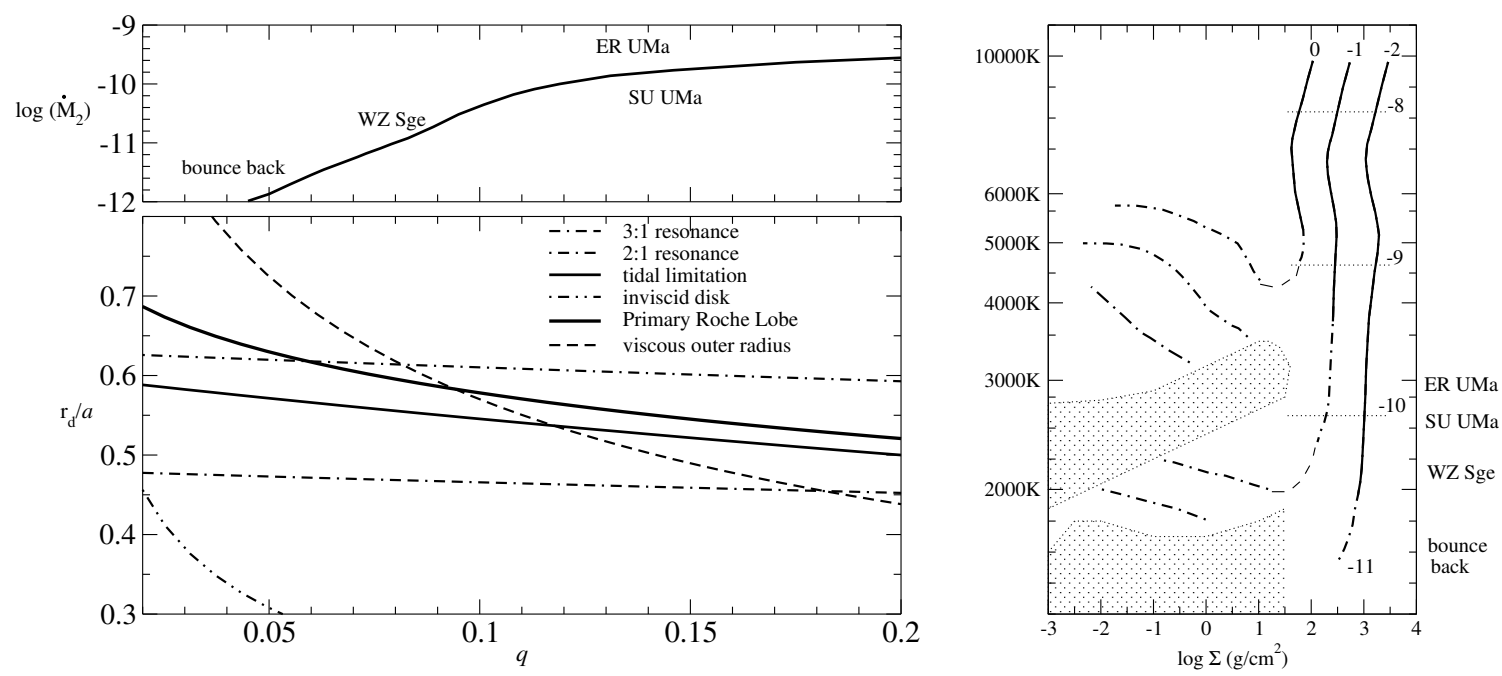

Figure 3: Left, top) The $\log (\dot{M})$ vs. mass ratio based on relationships from [12, 38]. Left, bottom) The characteristics radii of an accretion disk vs. mass ratio. Right) Slightly modified figure from [48] which illustrate the position of different types of short period cataclysmic variables at $\log \Sigma$ - Temperature - $\alpha$ $\dot{M}$ diagram. Marks corresponds to notifications described in [48], the solid lines shows optical thick disk solutions, and the dot-dashed lines show optical thin disks.

imum and are evolving back towards longer periods. After the binary has reached the period minimum, the mass-loss rate of the donor star drops by an order of magnitude [38]. From the observational point of view, these objects should be similar in appearance to the WZ Sge-type stars in quiescence. The compilation of bounce back candidates are presented in Table 2. All those systems have mass ratios less of 0.08. Similar to WZ Sge, the bounce back candidates do not show normal outbursts. Optical light curves in high inclination bounce back candidates demonstrate permanently double-humped light curves (marked by " $+q "$ in Table 2) more probably caused by the 2:1 resonance in its accretion disks (see Fig. 2). An identification of bounce-back systems is based on deriving the properties of the donor star which must be a brown-dwarf. Direct (spectral) evidence for brown-dwarf secondaries exists only for very few CVs (SDSS J143317.78+101123.3 and WZ Sge [39, 40]), whereas the recognition of other candidates is based on radial velocity studies and analysis of the spectral energy distribution. Using such indirect methods, solid evidence for very low mass donor stars was found for GD 552 [41], EZ Lyn [42], V406 Vir [43], SSS J122221.7-311525 [37].

\section{Accretion disk structure}

The accretion disk is a dominant source of radiation in SU UMa / ER UMa-type systems. However, the slope of optical spectra is not well described by the standard disk theory. The eclipsing mapping also shows a deviation from the expected temperature distribution of the steady- state accretion disk $[44,45]$. With the orbital period decreasing with time, the mass transfer rate falls and the accretion disk contribution to the total flux is diminished, as evident from clearly seen absorption lines of the WD in many WZ Sge-type objects. The theory predicts that accretion disks in CVs with mass transfer rates below $\sim 10^{-8} \mathrm{M}_{\odot} \mathrm{yr}^{-1}$ develop an optically thin outer region. This region begins to play a more important role in radiation with increasing the disk radius (see [46, 47, 48]). 
The study of the vertical structure of steady-state $\alpha$-disks shows two states with a high $(\sim 5000 \mathrm{~K})$ and a low $(\sim 2000 \mathrm{~K})$ temperature (see [48] and Figure 3, right). For $\alpha>0.1$ the warm solution is possible in the inner region of the accretion disk, however, at the larger disk radii, the material is in the cold state with $T \lesssim 3000 \mathrm{~K}$. Only the low-temperature state exists for $\alpha \approx 0.1$. When $\alpha$ decreases with temperature, this tendency to develop cold solutions in quiescence in enhanced.

The inner parts of the disk in SU UMa / ER UMa-type systems are usually optically thick and contribute to form the continuum. The contribution of disks to the continuum in WZ Sgetype and bounce-back systems is much less and does not exceed $\sim 40-50$ per cent in the optical range [43]. Various authors support an idea that the inner parts of the disk need to be cleared up during quiescence. It was invited to explain the long recurrent time of superoutbursts of WZ Sge and its behaviour in quiescence (see [33] and reference therein). The appearance of a cavity in the innermost parts of the disk can be caused by evaporation [49] or by the presence of the WD magnetic field [50].

The accretion disks in short period CVs often exhibit an azimuthal non-uniformity. One of the most mysterious observed emission structures is a bright spot on the leading side of the disk, opposite to the usual stream/disk impact position. This spot was found in HT Cas, VW Hyi, 1RXS J180834.7+101041, V406 Vir and many other short period CVs (see [52] and references therein). The statistical analysis of a sample of $68 \mathrm{CVs}$ with published emission line profiles showed that the presence of such a structure is a frequent phenomenon [51]. It was found that this emission region in HT Cas and other CVs is observed at the very edge of the accretion disk, which radius is close to the tidal truncation radius [52]. Neustroev et al. (2016) proposed that the leading side bright spot is caused by irradiation of tidally thickened sectors of the outer disk. We note that the normal hot spot and the emission structure on the opposite side of the disk can possibly explain double-humped light curves observed in WZ Sge-type stars [43, 53].

\section{Open questions}

There are a number of open questions. Some form them are: the origin of long periods no related with orbital periods in FS Aur, GW Lib and V455 And (see [54] and references therein); a source of unexplained quasi-periodic mini-outbursts in EZ Lyn [42] and V406 Vir [43]; the relatively short time (4 years) between superoutbursts in WZ-type system V406 Vir [43]; unusual long lasting super-outburst in WZ-Sge-type system SSS J122221.7-311523 [37].

\section{References}

[1] Kolb, U., \& Baraffe, I. 1999, MNRAS, 309, 1034

[2] Paczynski, B., \& Sienkiewicz, R. 1981, ApJ, 248, L27

[3] Gänsicke, B. T., Dillon, M., Southworth, J., et al. 2009, MNRAS, 397, 2170

[4] Zorotovic, M., Schreiber, M. R., Gansicke, B. T. 2011, A\&A, 536, A42

[5] Littlefair, S. P., Dhillon, V. S., Marsh, T. R., et al. 2008, MNRAS, 388, 1582

[6] Savoury, C. D. J., Littlefair, S. P., Dhillon, V. S., et al. 2011, MNRAS, 415, 2025

[7] Politano, M. 1996, ApJ, 465, 338 
[8] Wijnen, T. P. G., Zorotovic, M., \& Schreiber, M. R. 2015, A\&A, 577, A143

[9] Sion, E. M., \& Godon, P. 2012, Mem. Soc. Astron. Italiana, 83, 539

[10] Sion, E. M. 1995, ApJ, 438, 876

[11] Kato, T., Dubovsky, P. A., Kudzej, I., et al. 2014, PASJ, 66, 90

[12] Knigge, C., Baraffe, I., \& Patterson, J. 2011, ApJS, 194, 28

[13] Harrison, T. E., Bornak, J., Howell, S. B., et al. 2009, AJ, 137, 4061

[14] Thorstensen, J. R., Fenton, W. H., Patterson, J., et al. 2002, PASP, 114, 1117

[15] Thorstensen, J. R. 2013, PASP, 125, 506

[16] Kato, T., Imada, A., Uemura, M., et al. 2009, PASJ, 61, 395

[17] Warner, B. 1995, Cambridge Astrophysics Series, 28,

[18] Kato, T., Hambsch, F.-J., Maehara, H., et al. 2013, PASJ, 65, 23

[19] Kato, T., Hambsch, F.-J., Dubovsky, P. A., et al. 2015, PASJ, 251

[20] Robertson, J. W., Honeycutt, R. K., \& Turner, G. W. 1995, PASP, 107, 443

[21] Kato, T., \& Kunjaya, C. 1995, PASJ, 47, 163

[22] Patterson, J., Uthas, H., Kemp, J., et al. 2013, MNRAS, 434, 1902

[23] Kato, T., Ishioka, R., Isogai, K., et al. 2016, PASJ, 68, 107

[24] Olech, A., Wisniewski, M., Zloczewski, K., et al. 2008, Acta Astron., 58, 131

[25] Howell, S. B., Hoard, D. W., Brinkworth, C., et al. 2008, ApJ, 685, 418

[26] Patterson, J., Masi, G., Richmond, M. W., et al. 2002, PASP, 114, 721

[27] Patterson, J., Richman, H., Kemp, J., \& Mukai, K. 1998, PASP, 110, 403

[28] Baba, H., Sadakane, K., Norimoto, Y., et al. 2002, PASJ, 54, L7

[29] Howell, S. B., Adamson, A., \& Steeghs, D. 2003, A\&A, 399, 219

[30] Skidmore, W., Mason, E., Howell, S. B., et al. 2000, MNRAS, 318, 429

[31] Mason, E., Skidmore, W., Howell, S. B., et al. 2000, MNRAS, 318, 440

[32] Howell, S. B., Harrison, T. E., \& Szkody, P. 2004, ApJ, 602, L49

[33] Kuulkers, E., Henden, A. A., Honeycutt, R. K., et al. 2011, A\&A, 528, A152

[34] Kato, T. 2015, PASJ, 67, 108

[35] Nakata, C., Kato, T., Nogami, D., et al. 2014, PASJ, 66, 116

[36] Kato, T., Monard, B., Hambsch, F.-J., Kiyota, S., \& Maehara, H. 2013, PASJ, 65, L11

[37] Neustroev, V. V., Marsh, T. R., Zharikov, S. V., et al. 2017, MNRAS, 467, 597

[38] Sirotkin, F. V., \& Kim, W.-T. 2010, ApJ, 721, 1356

[39] Hernández Santisteban, J. V., Knigge, C., Littlefair, S. P., et al. 2016, Nature, 533, 366

[40] Harrison, T. E. 2016, ApJ, 816, 4

[41] Unda-Sanzana, E., Marsh, T. R., Gänsicke, B. T., et al. 2008, MNRAS, 388, 889 
[42] Aviles, A., Zharikov, S., Tovmassian, G., et al. 2010, ApJ, 711, 389

[43] Zharikov, S., Tovmassian, G., Aviles, A., et al. 2013, A\&A, 549, A77

[44] Bíró, I. B. 2000, A\&A, 364, 573

[45] Vrielmann, S. 2001, Astrotomography, Indirect Imaging Methods in Observational Astronomy, 573, 332

[46] Williams, R. E. 1980, ApJ, 235, 939

[47] Tylenda, R. 1981, Acta Astron., 31, 127

[48] Cannizzo, J. K., \& Wheeler, J. C. 1984, ApJS, 55, 367

[49] Meyer, F., \& Meyer-Hofmeister, E. 1994, A\&A, 288, 175

[50] Matthews, O. M., Wheatley, P. J., Wynn, G. A., \& Truss, M. R. 2006, MNRAS, 372, 1593

[51] Tappert, C., \& Hanuschik, R. 2001, Astrotomography, Indirect Imaging Methods in Observational Astronomy, 573, 119

[52] Neustroev, V. V., Zharikov, S. V., \& Borisov, N. V. 2016, A\&A, 586, A10

[53] Tovmassian, G., \& Zharikov, S. 2013, IAU Symposium, 290, 149

[54] Neustroev, V. V., Tovmassian, G. H., Zharikov, S. V., \& Sjoberg, G. 2013, MNRAS, 432, 2596

\section{DISCUSSION}

MARINA ROMANOVA: You mentioned that there is an evidence of spiral waves in the disk. Please, describe in greater details.

SERGEY ZHARIKOV: Observed brightness distribution in Doppler tomograms in some short period CVs can be interpreted as the evidence of spiral wave in the disk. The formation of spiral waves in an accretion disk of a close binary was proposed by Lin \& Papaliouzou in 1979 and explored by various authors. Spiral wave related with 2:1 resonance can be found in systems with extremely low mass ratio $<0.1$. The WZ Sge-type systems and related them bounce back candidate are examples of such objects. 